\title{
- Airborne measurements of the spatial distribution - of aerosol chemical composition across Europe and evolution of the organic fraction: Supplementary material
}

\author{
W. T. Morgan ${ }^{1}$, J. D. Allan ${ }^{1,2}$, K. N. Bower ${ }^{1}$, E.J. Highwood ${ }^{3}$, \\ D. Liu ${ }^{1}$, G. R. McMeeking ${ }^{1}$, M. J. Northway ${ }^{3}$, P. I. Williams ${ }^{1,2}$, \\ R. Krejci ${ }^{4}$ and H. Coe ${ }^{1}$
}

1. Centre for Atmospheric Science, University of Manchester, Manchester, UK

2. National Centre for Atmospheric Science, University of Manchester, UK

3. Department of Meteorology, University of Reading, UK

4. Department of Applied Environmental Science, Atmospheric Science Unit, Stockholm University, Sweden

\section{Scope}

The supplementary material outlined in this document is provided in order to present the meteorological context of the flight operations and support the analysis techniques and data quantification steps outlined in the main paper. The meteorological fields corresponding to each flying period are presented and further information regarding the photochemical context of the operations is presented. Further details regarding the volume closure between the Aerosol Mass Spectrometer (AMS) and the Passive Cavity Aerosol Spectrometer Probe (PCASP) are discussed. Comparison of the estimated HOA with primary combustion tracers is included. The relationship between the fractional contribution of Low-Volatility Oxygenated Organic Aerosol (LV-OOA) to the organic mass and the normalised organic signal at $\mathrm{m} / \mathrm{z} 44$ is also shown. Further information is provided regarding the Positive Matrix Factorisation (PMF) analysis examples from the main text, as well as a summary of some PMF diagnostics for the whole dataset. The PMF analysis was performed using the tools presented by Ulbrich et al. (2009). 


\section{Meteorological summary}

Figs. S1 and S2 display the typical meteorological conditions prevalent during each period considered by the analysis. The periods are relatively consistent in terms of their transport patterns, with the air masses transporting pollution from continental Europe downwind to either the UK region or into the Eastern Atlantic Ocean. Thus the flights are predominantly focused upon either sampling such pollution over continental Europe itself or at a range of scales downwind.

The evolution of the aerosol chemical composition during the LONGREX-2 period was examined based upon the relatively consistent transport patterns prevalent during the period. Fig. S3a displays the back trajectories for each flight during this period based upon Straight and Level Runs (SLRs) during each flight. The trajectories display highly consistent behaviour during the period, which is unsurprising given the relative stability of the high pressure system located over Northern Europe during this period. Fig. S3b highlights the back trajectory from the 14 May 2008, which was initialised from a SLR during B374 in the Eastern Atlantic Ocean. B374 represented the endpoint in our operations during this period both in terms of the geographical location of the missions and also the distance from continental European sources i.e. the most aged polluted air mass. The back trajectory indicates that the spatial coverage of the flight operations closely matches the air mass transport during the period leading up to the 14 May 2008. Specifically, flights B370-B374 took place across Northern Europe during this period covering close to 5 days of air mass transport.

\section{Photochemical context}

The relationship between $\mathrm{O}_{3}$ and $\mathrm{CO}$ with the $\mathrm{O}_{3}: \mathrm{NO}_{x}$ ratio discussed in the main paper is presented in Fig. S4. The results indicate that $\mathrm{O}_{3}$ increases and $\mathrm{CO}$ decreases steadily in the 1-100 $\mathrm{O}_{3}: \mathrm{NO}_{x}$ range, which is a reflection of photochemistry and dilution respectively. Beyond an $\mathrm{O}_{3}: \mathrm{NO}_{x}$ ratio of 100, the concentrations decrease with $\mathrm{CO}$ returning to background levels and $\mathrm{O}_{3}$ remaining relatively constant in the 40-60 ppb range.

\section{HOA estimation and LV-OOA interpretation}

Included in Fig. S5a are correlations of the estimated HOA concentration with Black Carbon (BC), $\mathrm{NO}_{x}$ and $\mathrm{CO}$. These indicate that for 8 of the flights, the correlations of the estimated HOA with these primary emission tracers are greater than 0.5. Correlations lower than 0.5 are generally encountered on flights where these tracers and the estimated HOA are very low, thus the correlations break down at values when the relationships exhibit enhanced noise due to low signal. This is demonstrated in Fig. S5b and c, where at low concentrations the relationships are relatively flat but at enhanced concentrations, the correlation is greater. Given the simple nature of the HOA estimate, these correlations and relationships do provide some confidence that the estimated HOA provides a qualitative indicator of the contribution of HOA to the 
OM burden. Also shown in Fig. S5a are the correlations between the Low-Volatility Oxidised Organic Aerosol (LV-OOA) organic mass fraction and the m/z 44:OM ratio described in the main paper.

\section{AMS versus PCASP comparison}

Validation of the collection efficiency treatment applied to the dataset following the principles developed by Matthew et al. (2008) is accomplished by comparing the AMS data with the volume estimated concentrations from the PCASP instrument. The AMS total mass concentrations were converted to total volume concentrations using the densities reported by Cross et al. (2007), which correspond to $1.27 \mathrm{~g} \mathrm{~cm}^{-3}$ for organics and $1.77 \mathrm{~g} \mathrm{~cm}^{-3}$ for inorganics. A comparison of the estimated volume from the AMS and PCASP is shown for SLRs below $3000 \mathrm{~m}$ in Fig. S6. Over all of the considered flights, the estimated AMS volume concentrations were $26 \%$ higher than the estimated PCASP volumes. This average agreement is predominantly determined by the LONGREX flights, which were quite consistent in terms of the agreement from flight-toflight. The ADIENT flying periods sit on either side of the overall regression slope, with the ADIENT-2 flying indicating that the PCASP volume was $48 \%$ of the AMS volume. These discrepancies are considered tolerable given the large uncertainties previously reported in the literature for PCASP volume estimates (e.g. Moore et al., 2004; Hallar et al., 2006) and the uncertainties in the AMS volume estimates.

For B357, the PCASP volume estimate was more than two times greater than the AMS volume estimate, which is outside of the bounds of uncertainty for the two instruments. The reason for this discrepancy is unknown but could reflect an artifact in either instrument or the presence of material that is not detected by the AMS. The discrepancy between the two instruments is also reflected in the calculated volume-scattering relationship when comparing the measurements with a nephelometer system. The main difference between B357 and the other flights in the dataset is the sampling altitude of the aircraft, where in B357, the aircraft operated at a constant altitude of $200 \mathrm{~m}$ for the majority of the flight. The other flights in the dataset operated at altitudes higher in the boundary layer. Potentially, the nephelometer and PCASP measurements could be perturbed by the constant low-level flying in a humid environment as the aerosol sampled may not be sufficiently dried in the inlet lines and by the heater respectively. The AMS volume estimates do not include water, so this could potentially cause the discrepancy. Additionally, the PCASP and nephelometer may be measuring refractory material or particles above the cut off of the AMS aerodynamic lens. This would also lead to the AMS underestimating the volume relative to the PCASP.

\section{Additional organic factor analysis diagnostics}

A summary of the $Q / Q_{\text {expected }}$ parameter is shown in Fig. S7, which indicates that the parameter is generally less than 2 . Four flights had $Q / Q_{\text {expected }}$ values greater than 2, with the largest value being 5.01 (B374). Such values are greater than is generally considered optimal if attempting to produce a perfect characterisation of the dataset but 
given the difficulty in deriving robust results when more than 2 factors are chosen, it is not possible to reduce $\mathrm{Q}$ further. Consequently, the additional $\mathrm{Q}$ contribution prevalent in the dataset is considered as 'model error'.

\subsection{Example PMF solutions}

The time series, mass spectra and residuals for the two factor solutions for the three example flights used to describe the factor analysis in the main paper are presented here. B369 is also included as an additional example, reflecting background conditions for comparison with the more polluted examples. The results for B357 are shown in Fig. S8, for B362 in Fig. S9, for B369 in Fig. S10 and B406 in Fig, S11. All the examples show excellent agreement between the measured and reconstructed organic mass concentrations.

\subsection{Bootstrapping analysis}

Numerical stability of the 2-factor solutions was quantitatively evaluated using a bootstrapping analysis (Ulbrich et al., 2009, and references therein) where random resampling of the data matrix is performed in the time dimension. This analysis was performed using 20 iterations, with the results being grouped according to the uncentred correlation coefficient between mass spectral profiles. The results from the bootstrapping analysis are shown for B357 in Fig. S12, for B362 in Fig. S13, for B369 in Fig. S14 and for B406 in Fig. S15. The bootstrapping results for all the flights are summarised in Table S1. The variance of the solutions in both the time series and factor profile dimensions is evaluated using suitable metrics. The time series diagnostic is the mean of the standard deviation for each factor, reported as a percentage of the overall mean mass concentration. For the mass spectra, the greatest standard deviation for each factor profile from the bootstrapping analysis is reported. A mean is not deemed appropriate to evaluate the stability of the mass spectra as the chemical assignment of factors is performed based on a limited number of peaks (i.e. less than 10).

The OOA-1 (LV-OOA) factor profiles are highly robust with little deviation between the average mass spectrum from the bootstrapping analysis and the base solution. Furthermore, the standard deviations are typically low. This is a consistent result throughout the dataset, which is shown by the low scores for the diagnostics in Table S1. The OOA-2 factor profiles are more variable for B362, B369 and B406, with less stability in the signal intensity at $\mathrm{m} / \mathrm{z} 44$. This reflects the continuum nature of the $\mathrm{OM}$ discussed in the main text, whereby there is significant variability on a given flight in the level of oxidation. Thus by randomly resampling the dataset in the time dimension using the bootstrapping procedure, conditions with either enhanced or diminished $\mathrm{m} / \mathrm{z}$ 44 in the OOA-2 may be encountered and this is reflected by enhanced standard deviations in both the time series and mass spectra. The normalised standard deviation for the time series of OOA-2 for B369 is much greater than the other flights in the dataset. This is predominantly a result of the low concentrations encountered during the flight, coupled with large standard deviations which are associated with large changes in the composition of the OOA-2 component in this instance. For much of the flight, signal at $\mathrm{m} / \mathrm{z} 57$ is close to zero indicating that HOA makes a minimal contribution to the 
147 OOA-2 component. However, during the peak OM event at 15:47 UTC, signal at $\mathrm{m} / \mathrm{z}$ 14857 is enhanced and thus the contribution of HOA is likely elevated. This event co149 incides with the maximum in the $\mathrm{NO}_{x}$ concentration and is associated with potential 150 sampling of low-level urban outflow from Stockholm into the Baltic Sea. During this 151 period, the standard deviations for the OOA-2 factor increase. Thus such changes in 152 the OOA-2 composition from SV-OOA dominated to HOA dominated are reflected by 153 large increases in the standard deviation values from the bootstrapping analysis. The 154 much lower normalised standard deviation values associated with the time series of 155 the OOA-2 components for the rest of the dataset suggest this feature to be atypical.

156 This is a reflection of the regional nature of the measurements, with few instances of 157 prolonged exposure to intense urban signatures.

158 The results presented here demonstrate the robustness of the chosen 2-factor solu159 tions in terms of both the mass spectra and time series of the components. The OOA-1

160 (LV-OOA) components are highly numerically stable, while the decreased numerical 161 stability of the OOA-2 (SV-OOA and HOA) components is entirely consistent with the 162 continuum of oxidation/aging discussed in the main text. 


\section{References}

Cross, E. S., Slowik, J. G., Davidovits, P., Allan, J. D., Worsnop, D. R., Jayne, J. T., Lewis, D. K., Canagaratna, M., and Onasch, T. B.: Laboratory and Ambient Particle Density Determinations using Light Scattering in Conjunction with Aerosol Mass Spectrometry, Aerosol Science and Technology, 41, 343-359, URL http: //www . informaworld.com/10 .1080/02786820701199736, 2007.

Hallar, A. G., Strawa, A. W., Schmid, B., Andrews, E., Ogren, J., Sheridan, P., Ferrare, R., Covert, D., Elleman, R., Jonsson, H., Bokarius, K., and Luu, A.: Atmospheric Radiation Measurements Aerosol Intensive Operating Period: Comparison of aerosol scattering during coordinated flights, J. Geophys. Res., 111, -, URL http://dx.doi .org/10.1029/2005JD006250, 2006.

Kirchstetter, T. W., Harley, R. A., Kreisberg, N. M., Stolzenburg, M. R., and Hering, S. V.: On-road measurement of fine particle and nitrogen oxide emissions from lightand heavy-duty motor vehicles, Atmospheric Environment, 33, 2955-2968, 1999.

Matthew, B. M., Middlebrook, A. M., and Onasch, T. B.: Collection efficiencies in an Aerodyne Aerosol Mass Spectrometer as a function of particle phase for laboratory generated aerosols, Aerosol Science and Technology, 42, 884-898, 2008.

Moore, K. G., I., Clarke, A. D., Kapustin, V. N., McNaughton, C., Anderson, B. E., Winstead, E. L., Weber, R., Ma, Y., Lee, Y. N., Talbot, R., Dibb, J., Anderson, T., Doherty, S., Covert, D., and Rogers, D.: A comparison of similar aerosol measurements made on the NASA P3-B, DC-8, and NSF C-130 aircraft during TRACE-P and ACE-Asia, J. Geophys. Res., 109, -, URL http://dx.doi.org/10.1029/ 2003JD003543, 2004.

Ulbrich, I. M., Canagaratna, M. R., Zhang, Q., Worsnop, D. R., and Jimenez, J. L.: Interpretation of organic components from Positive Matrix Factorization of aerosol mass spectrometric data, Atmospheric Chemistry and Physics, 9, 2891-2918, 2009. 
Table S1: Summary of the diagnostics relating to the bootstrapping analysis from the dataset for each flight.

\begin{tabular}{ccccc}
\hline Flight & \multicolumn{2}{c}{ SD $_{t s} /$ TS $(\%)$} & \multicolumn{2}{c}{ Max $\left(\mathrm{SD}_{m s}\right)(\%)$} \\
& OOA-1 & OOA-2 & OOA-1 & OOA-2 \\
\hline B357 & 2.0 & 2.5 & 0.77 & 0.85 \\
B362 & 5.6 & 9.7 & 1.00 & 2.44 \\
B365 & 2.8 & 3.0 & 0.62 & 1.31 \\
B366 & 13.4 & 11.8 & 1.66 & 1.46 \\
B369 & 19.1 & 45.9 & 2.23 & 1.66 \\
B370 & 2.9 & 3.6 & 0.52 & 0.46 \\
B371 & 4.8 & 8.0 & 0.41 & 1.82 \\
B373 & 2.3 & 3.9 & 1.06 & 0.86 \\
B374 & 1.1 & 1.7 & 0.44 & 0.68 \\
B379 & 6.6 & 9.3 & 0.61 & 1.63 \\
B380 & 1.4 & 2.4 & 0.36 & 0.37 \\
B401 & 11.1 & 13.3 & 0.24 & 0.32 \\
B402 & 5.5 & 14.9 & 0.76 & 1.23 \\
B406 & 1.4 & 1.8 & 1.06 & 0.79 \\
\hline
\end{tabular}



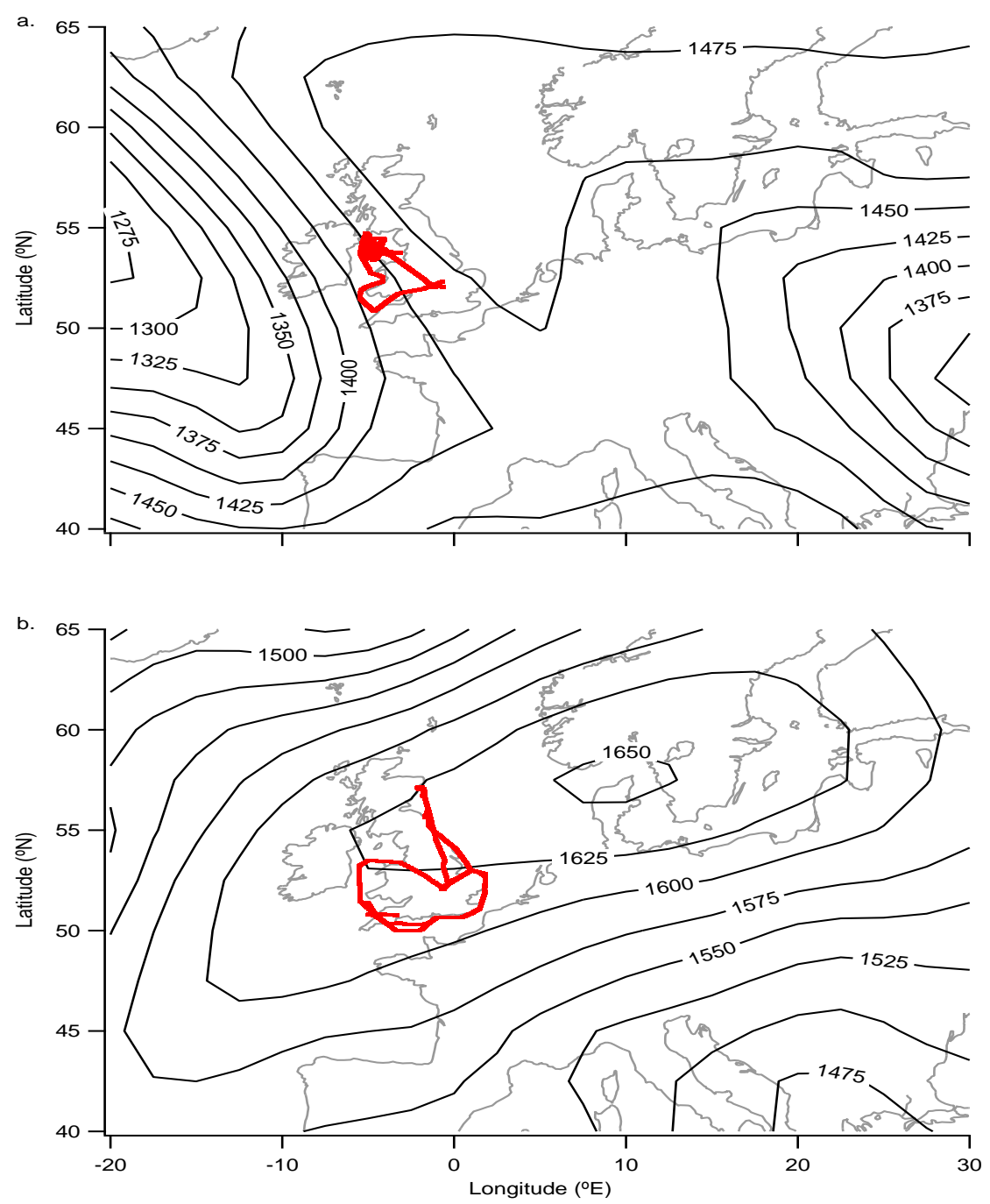

Figure S1: Flight tracks of the BAe-146 considered by this analysis for the ADIENT periods. Also shown are ECMWF $850 \mathrm{hPa}$ geopotential height fields for each period considered by the analysis, where the field is either pertinent to a particular flight or is representative of the overall meteorological regime of the period. All geopotential height fields are for 12UTC. (a) summarises the flights for the UK-based ADIENT flying in April 2008 and the geopotential height field is from 16 April 2008 (B357). (b) summarises the flights for the UK-based ADIENT flying in September 2008 and the geopotential height field is from 25 September 2008 (B406). 

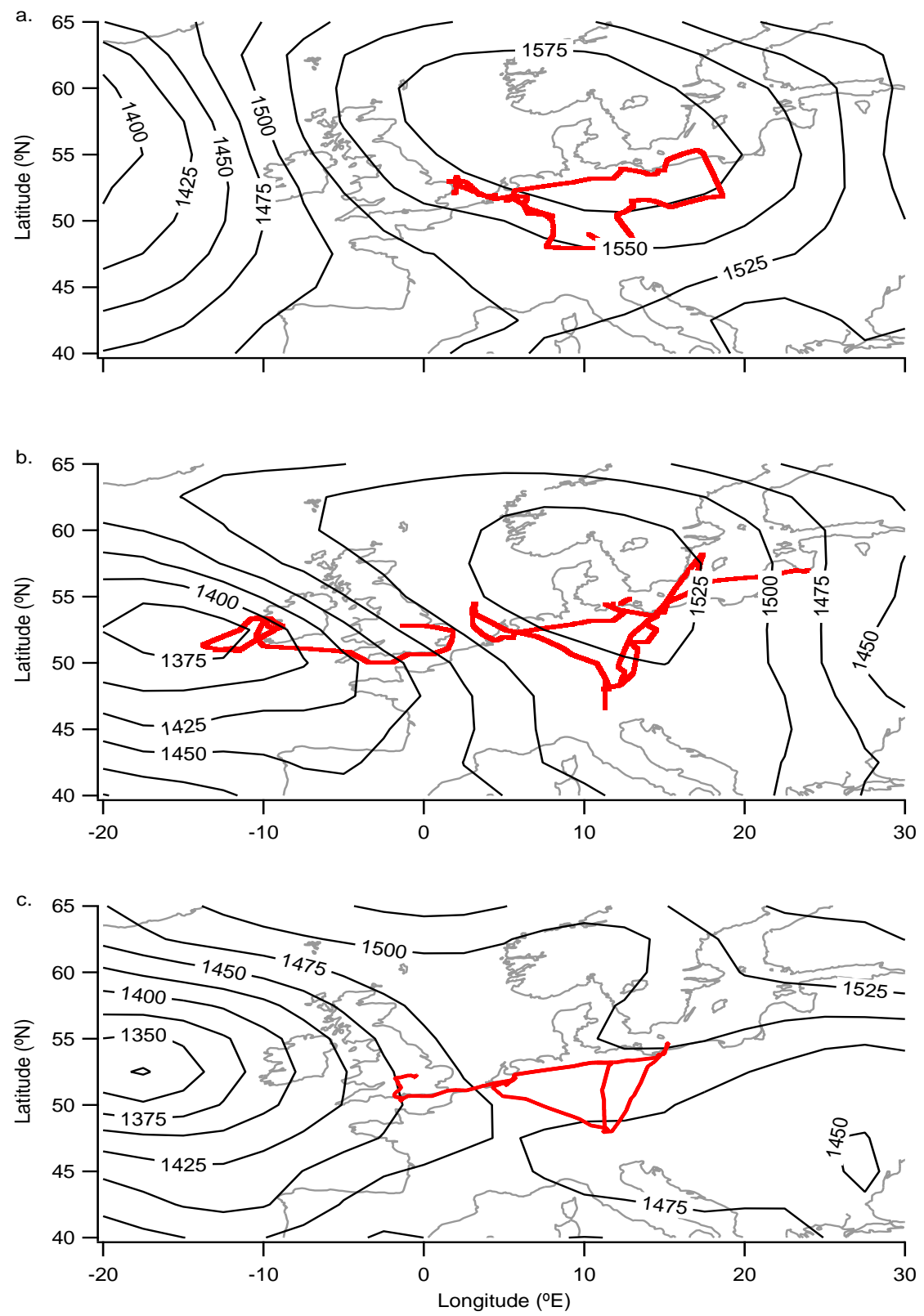

Figure S2: Same plots as Fig. S1 but now for the LONGREX flying period. (a) summarises the flights for the LONGREX-1 period with a geopotential height field for the 06 May 2008. (b) summarises the flights for the LONGREX-2 period with a geopotential height field for the 14 May 2008. (c) summarises the flights for the LONGREX-3 period with a geopotential height field for the 22 May 2008. 

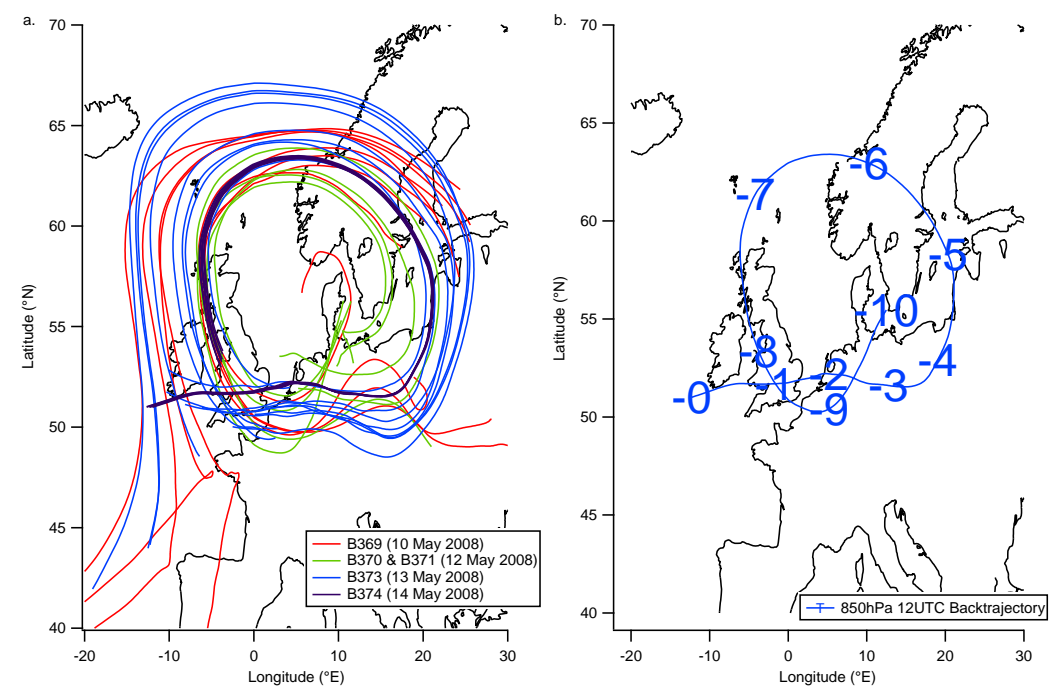

Figure S3: (a) Back trajectories at $850 \mathrm{hPa}$ initialised from $1200 \mathrm{UTC}$ on each flight day from the LONGREX-2 period. The initialisation points correspond to several SLRs during each flight. (b) Air mass back trajectory initialised from 1200 UTC on 14 May 2008 at $850 \mathrm{hPa}$. The numbered points relate to the number of days passed since the air mass was in that location. 

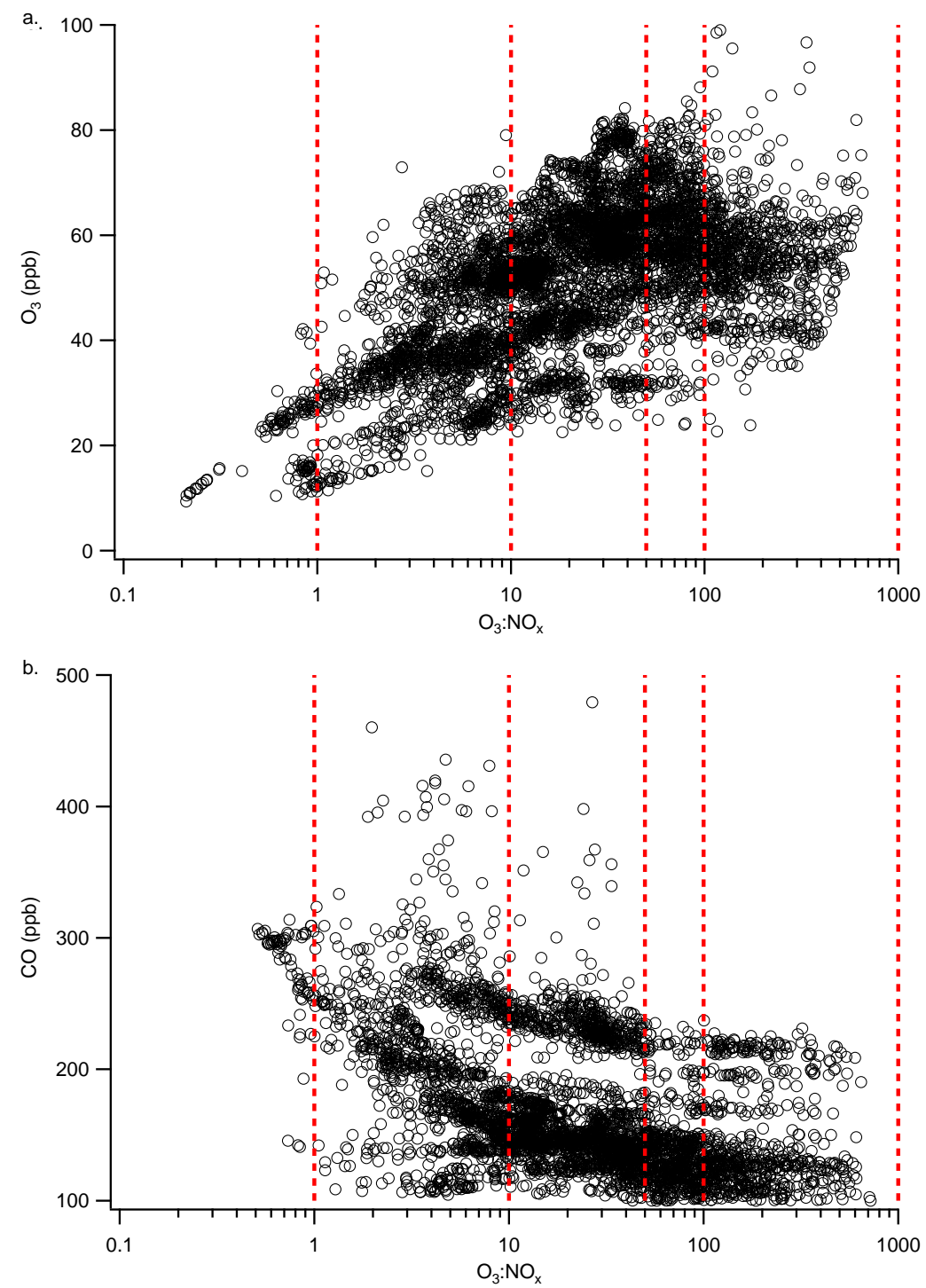

Figure S4: (a) Relationship between $\mathrm{O}_{3}$ and $\mathrm{O}_{3}: \mathrm{NO}_{x}$ for all flights. (b) Relationship between $\mathrm{CO}$ and $\mathrm{O}_{3}: \mathrm{NO}_{x}$ for all flights expect for ADIENT-2. The red dashed lines refer to the distance from source boundaries discussed in the main paper. 
a.

HOA - BC $\square$ HOA - NO $\square$ HOA - CO $\square$ LV-OOA - m/z 44:OM

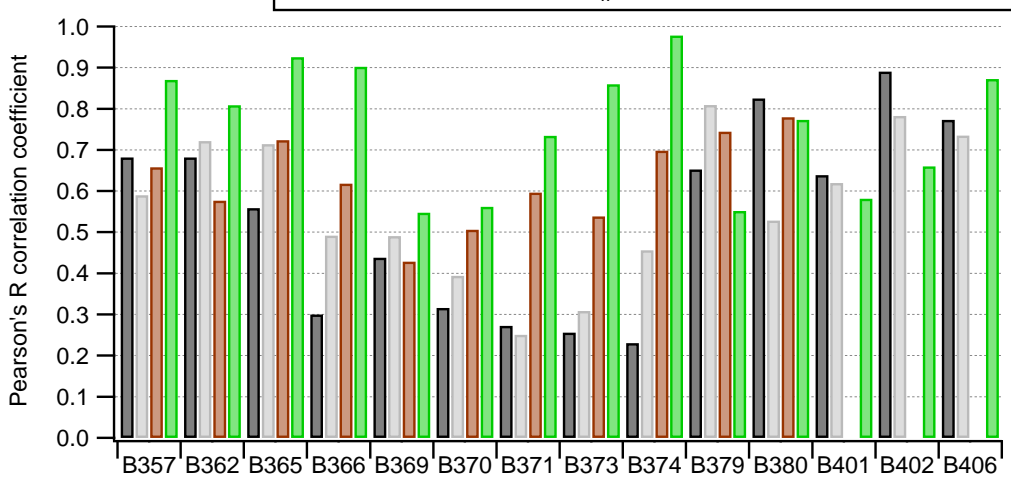

Flight Number
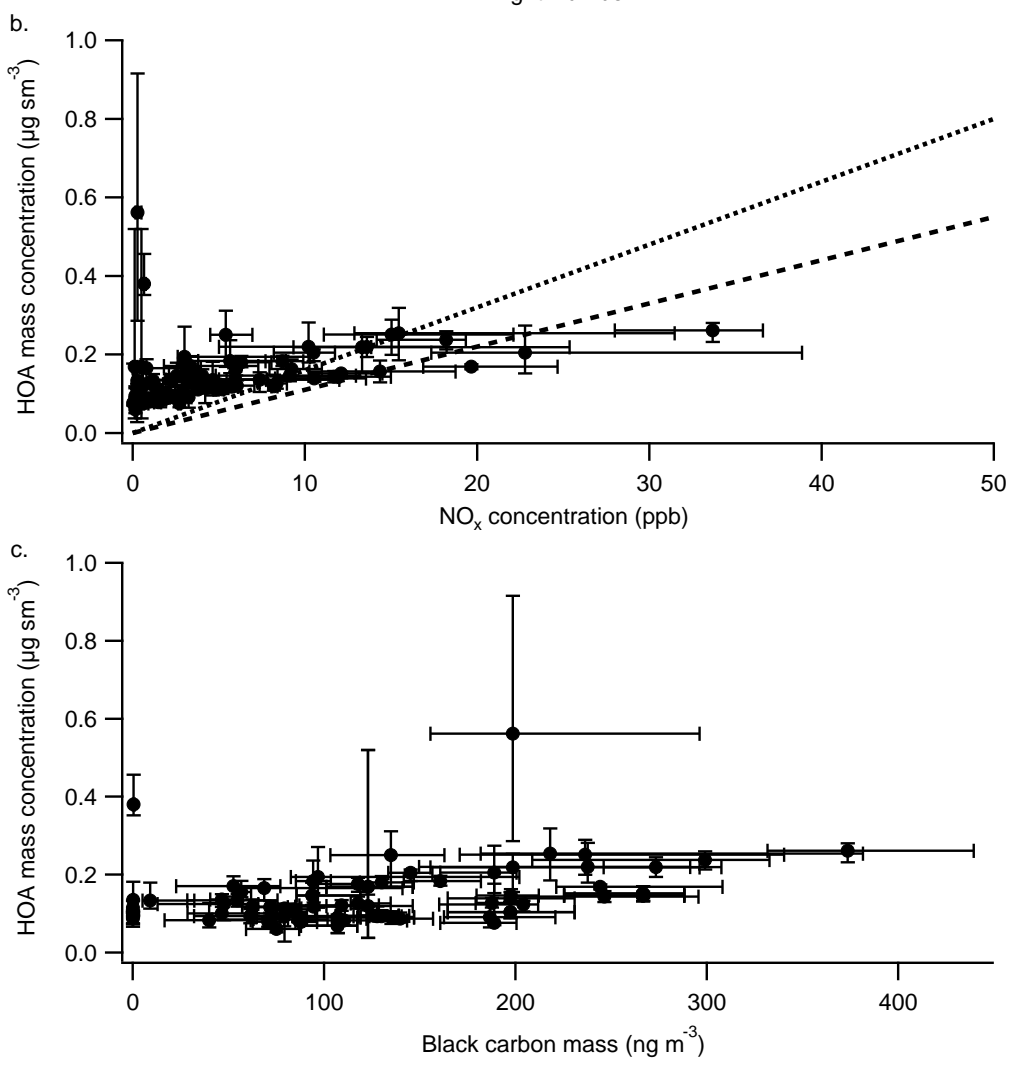

Figure S5: (a) Summary of correlations for estimated HOA with Black Carbon (BC), $\mathrm{NO}_{x}$ and CO. Also shown are the correlations for LV-OOA organic mass fractions with the normalised organic signal at $\mathrm{m} / \mathrm{z} 44$. (b) and (c) display the median estimated HOA versus $\mathrm{NO}_{x}$ and $\mathrm{BC}$ respectively for SLRs across the whole dataset. The error bars reflect the $25^{\text {th }}$ and $75^{\text {th }}$ percentiles on each SLR. The black dotted lines in (b) correspond to the literate POA: $\mathrm{NO}_{x}$ ratios reported by Kirchstetter et al. (1999). 


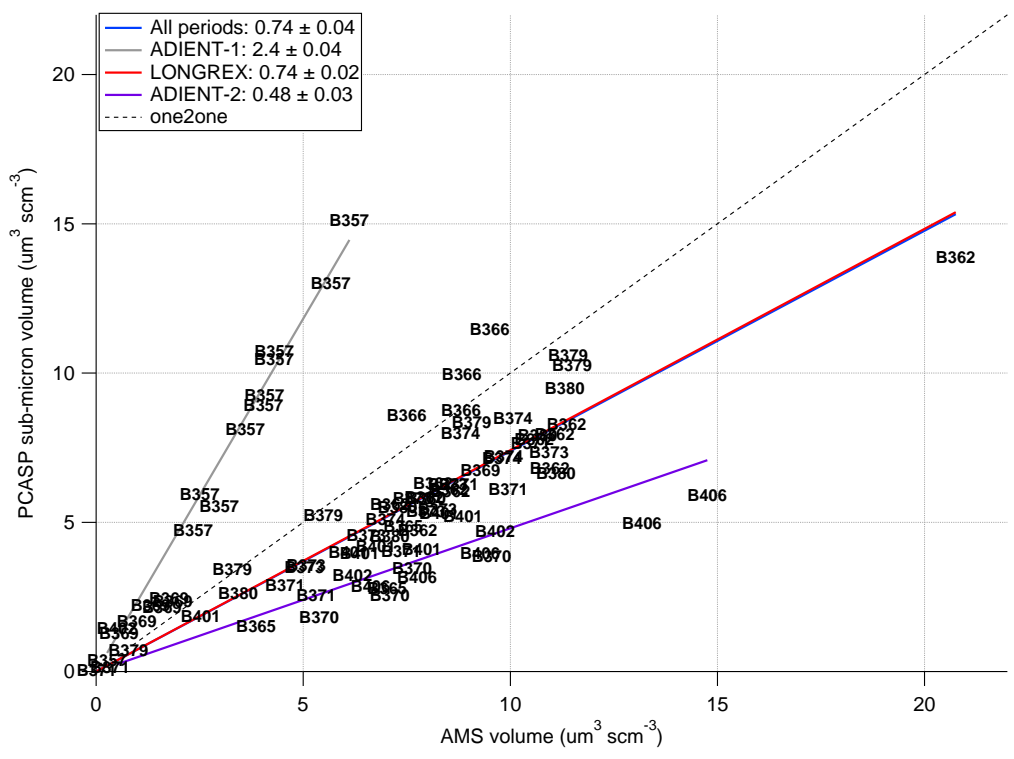

Figure S6: Comparison of inferred volume from the AMS with estimated sub-micron volume derived from the PCASP. The markers refer to SLRs below $3000 \mathrm{~m}$ i.e. within the boundary layer. The text markers refer to the flight which the point is from. Linear regression lines are shown for both individual flying periods and the study as a whole.

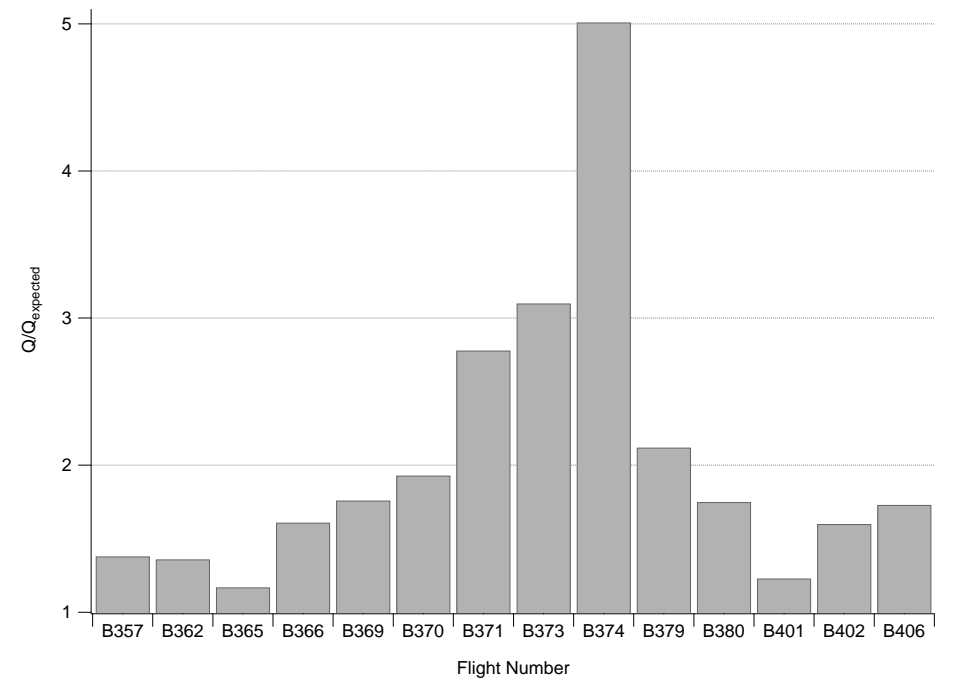

Figure S7: Summary of the $Q / Q_{\text {expected }}$ parameter for each flight in the dataset. All values are for the two-factor solutions with an fPeak of zero. 

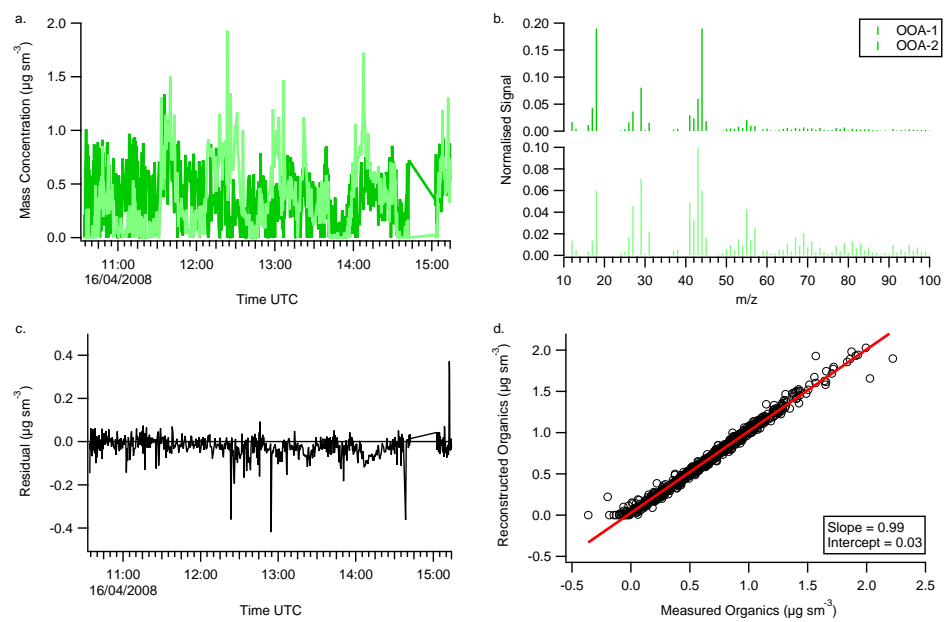

Figure S8: PMF solution for the two factor case from flight B357 including factor component time series (a) and mass spectra (b). The absolute residual is also shown in (c), whilst a comparison between the factor analysis reconstructed mass and measured organic signal is displayed in (d).
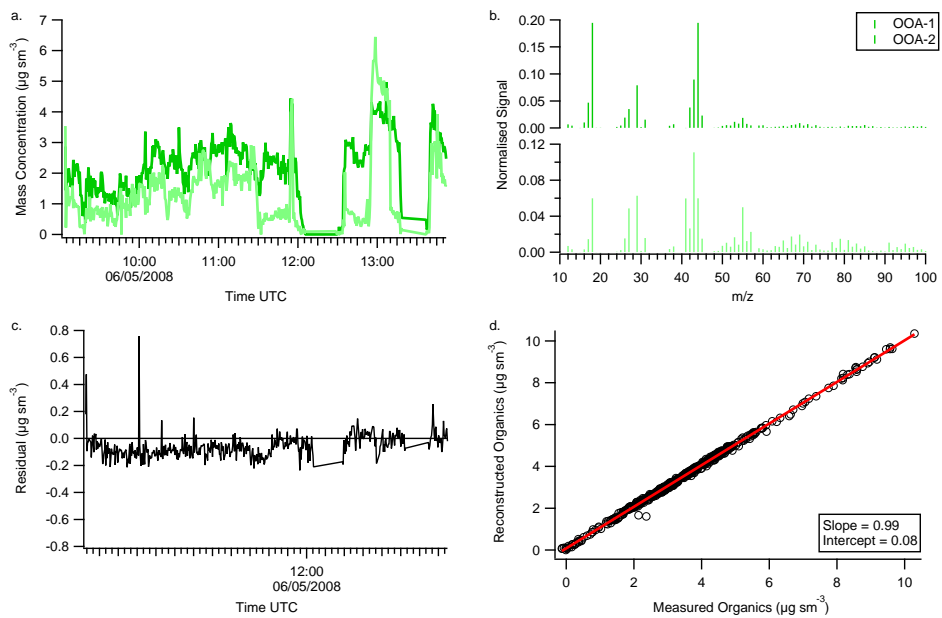

Figure S9: PMF solution for the two factor case from flight B362 including factor component time series (a) and mass spectra (b). The absolute residual is also shown in (c), whilst a comparison between the factor analysis reconstructed mass and measured organic signal is displayed in (d). 

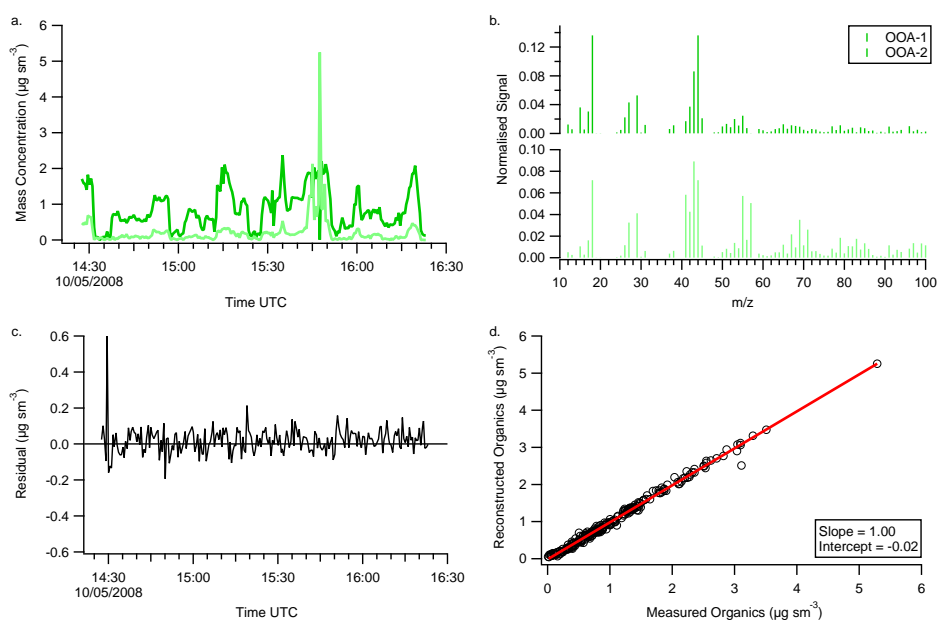

Figure S10: PMF solution for the two factor case from flight B369 including factor component time series (a) and mass spectra (b). The absolute residual is also shown in (c), whilst a comparison between the factor analysis reconstructed mass and measured organic signal is displayed in (d).
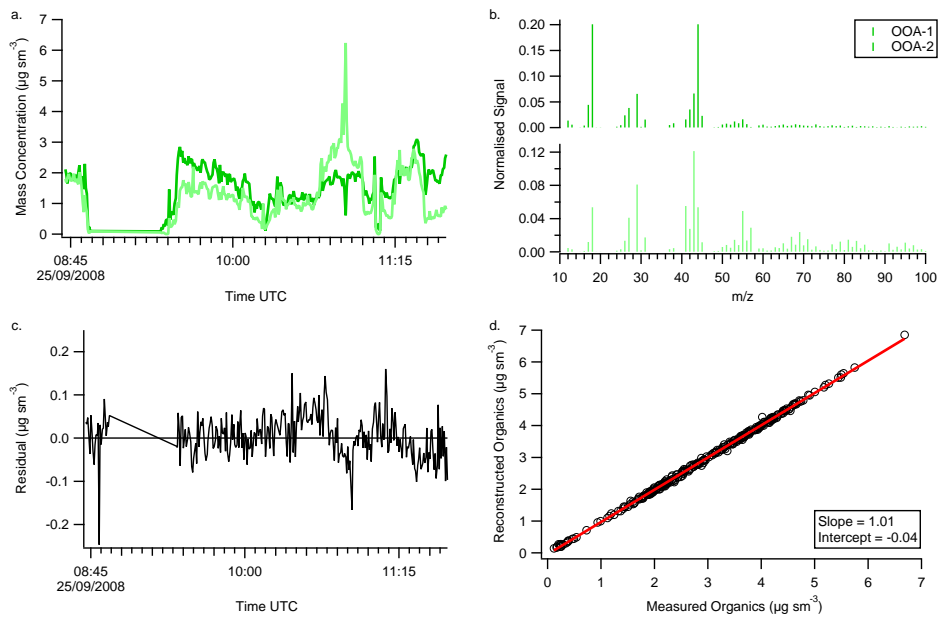

Figure S11: PMF solution for the two factor case from flight B406 including factor component time series (a) and mass spectra (b). The absolute residual is also shown in (c), whilst a comparison between the factor analysis reconstructed mass and measured organic signal is displayed in (d). 

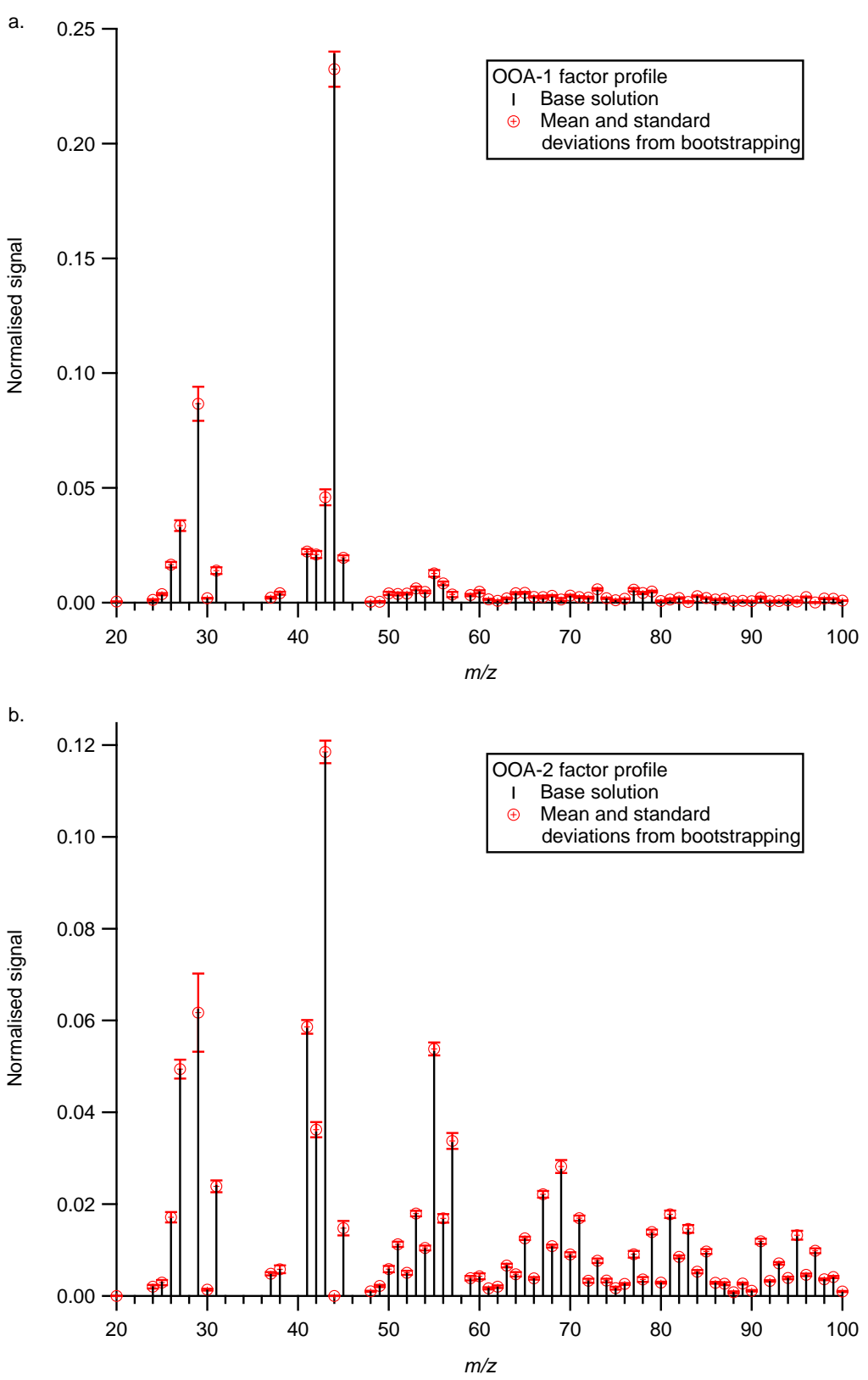

Figure S12: Results of the bootstrapping analysis for the two factor solution mass spectra for flight B357. (a) displays the mass spectrum for OOA-1, while (b) displays the mass spectrum for OOA-2. 

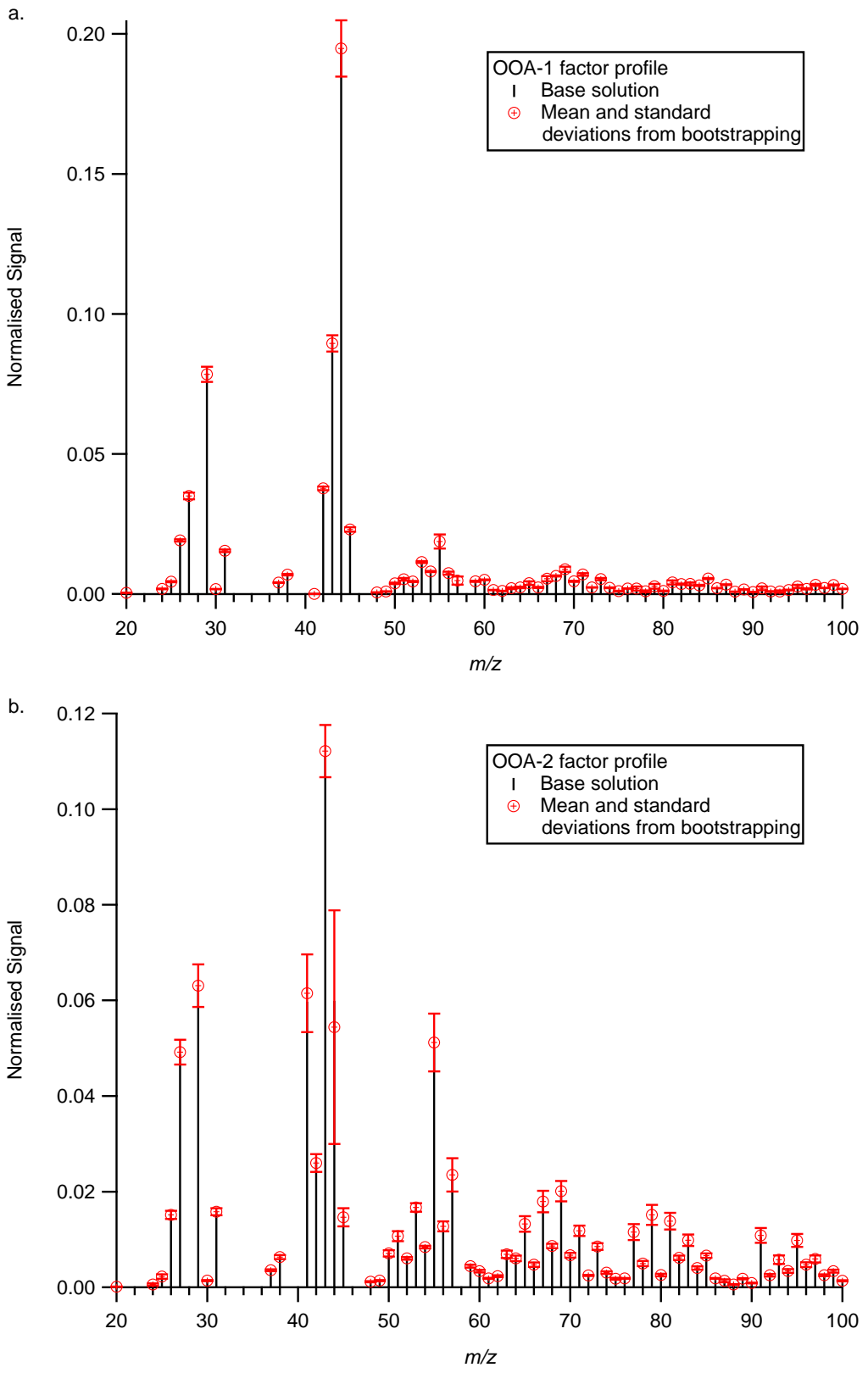

Figure S13: Results of the bootstrapping analysis for the two factor solution mass spectra for flight B362. (a) displays the mass spectrum for OOA-1, while (b) displays the mass spectrum for OOA-2. 

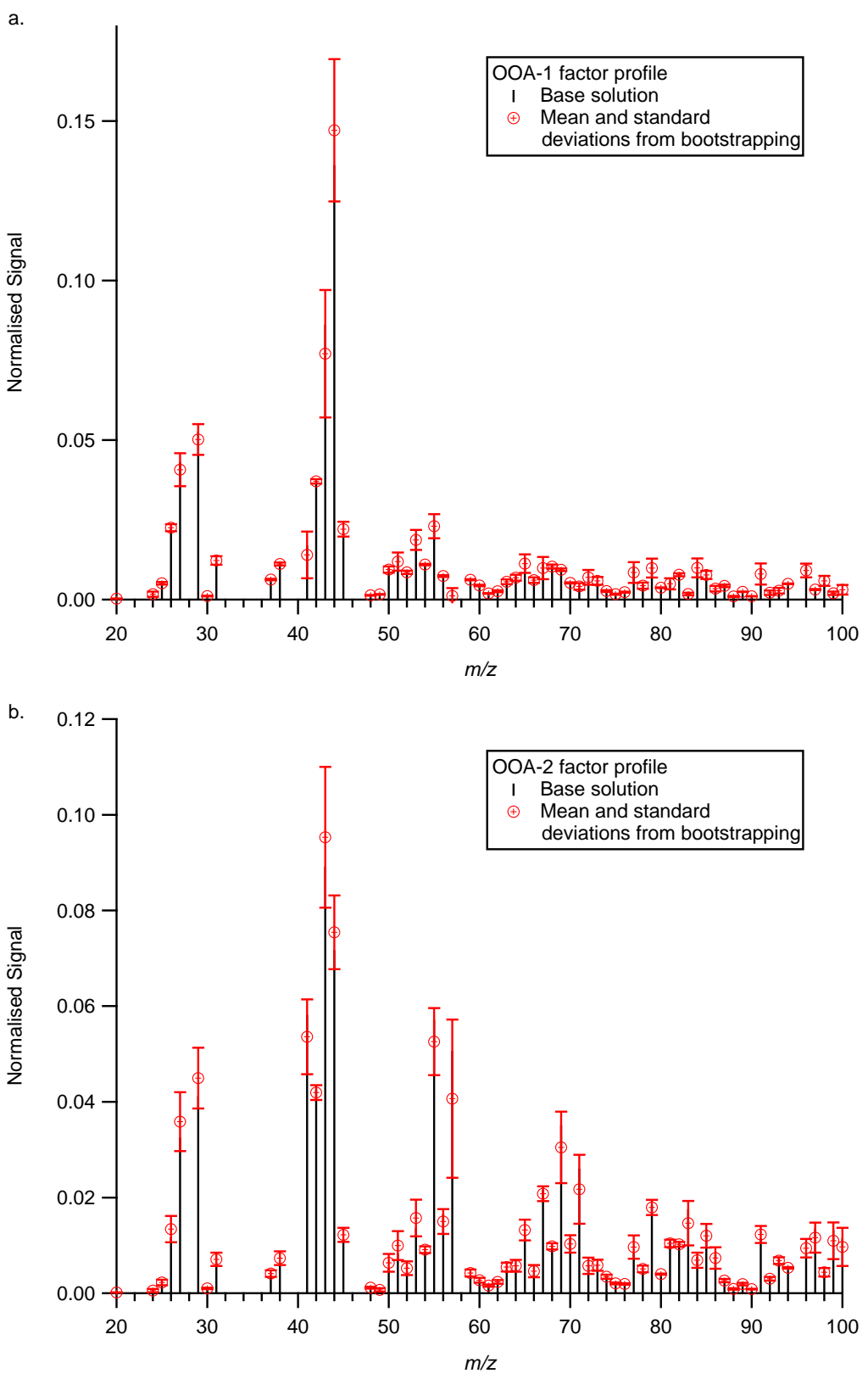

Figure S14: Results of the bootstrapping analysis for the two factor solution mass spectra for flight B369. (a) displays the mass spectrum for OOA-1, while (b) displays the mass spectrum for OOA-2. 

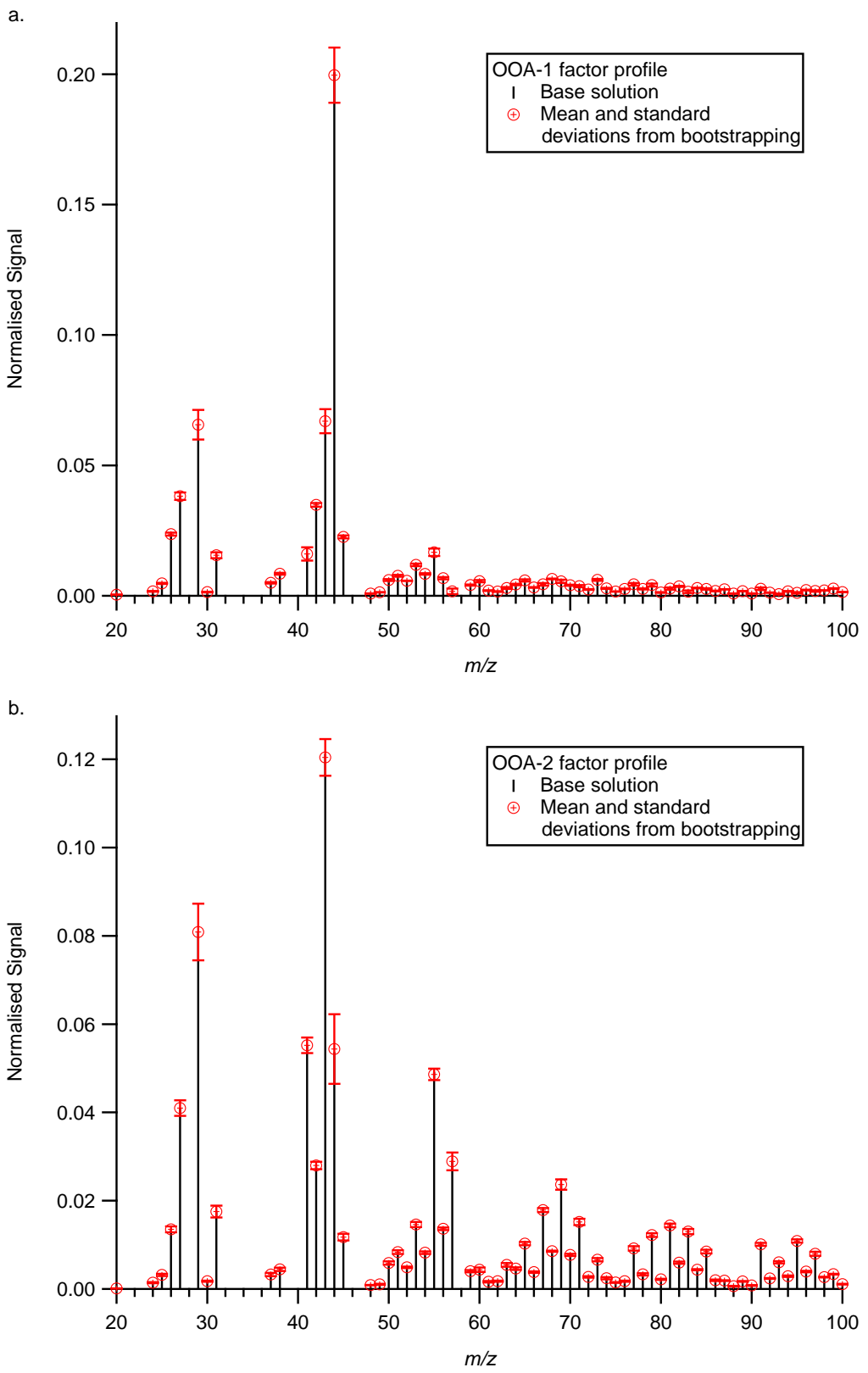

Figure S15: Results of the bootstrapping analysis for the two factor solution mass spectra for flight B406. (a) displays the mass spectrum for OOA-1, while (b) displays the mass spectrum for OOA-2. 